\title{
Stress Diastolic Function for Assessment of Coronary Artery Disease
}

\author{
Naveen Kumar Murki ${ }^{1}$ Jeethender Kumar Jain Kala \\ ${ }^{1}$ Department of Cardiology, Nizam's Institute of Medical Sciences, \\ Punjagutta, Hyderabad, Telangana, India \\ 2Department of Obstetrics \& Gynaecology, NICE Hospital, \\ Masab Tank, Hyderabad, Telangana, India \\ Ind J Car Dis Wom 2019;4:20-25
}

Mythri Mamidi²

\begin{abstract}
Address for correspondence Naveen Kumar Murki, MD, DM, Department of Cardiology, Nizam's Institute of Medical Sciences, Punjagutta, Hyderabad 500081, Telangana, India (e-mail: naveenjust@ gmail.com).
\end{abstract}

\begin{abstract}
Keywords

- coronary artery disease

- diastolic dysfunction

- stress echo

Introduction Prevalence of coronary artery disease (CAD) has increased to alarming levels globally. There is a significant improvement in the noninvasive modalities for the early CAD detection by costly investigations such as computed tomographic (CT) coronary angiogram. The authors want to see the efficacy of adding pulse and tissue Doppler parameters, which is a cheaper modality, to the TMT (treadmill test) stress testing, for the detection of CAD.

Materials and Methods The authors have recruited 51 patients, who were referred for TMT for detection of inducible ischemia. Mean age of the patients was 51.9 years. Patients with renal failure, rhythm disturbances, and hypertension more than stage 1 were excluded. Along with the TMT, basal and exercise pulse wave and tissue Doppler parameters were recorded. These echocardiogram parameters were compared with presence and absence of CAD, along with the severity of CAD (SVD [single-vessel disease], DVD [double-vessel disease], and TVD [triple-vessel disease]) on a conventional coronary angiogram (CAG).

There was no statistical difference between basal and after TMT in pulse wave diastolic parameters (basal E-wave velocities: $0.76 \pm 0.18$ vs. $0.88 \pm 0.22, p=0.29$ : after TMT: $1.03 \pm 0.22$ vs. $1.12 \pm 0.21, p=0.29$ ) for detection of CAD on CAG. However, most tissue Doppler velocities were significantly different both in basal and after TMT in CAD group when compared with no-CAD group ( $S^{\prime}$ septal, basal, and after TMT, $p=0.002$, $\mathrm{E}^{\prime}$ septal, basal, and after TMT, $p=0.0015, \mathrm{~A}^{\prime}$ septal, basal, and after TMT, $p=0.0195$, $S^{\prime}$ lateral, basal, and after TMT, $p=0.0018$, $E^{\prime}$ lateral, basal, and after TMT, $p=0.0016$, $S^{\prime}$ Inferior, basal, and after TMT, $p=0.0036, A^{\prime}$ inferior, basal, and after TMT, $p=0.023$ ). Average $E / E^{\prime}$ after TMT for DVD was $12.29 \pm 2.09$ (vs. no-CAD $10.70 \pm 2.00$ ) and for TVD it was $12.2 \pm 2.19$ (vs. no-CAD $10.69 \pm 2.1$ ), which were statistically significantly higher than no-CAD ( $p=0.02, p=0.02)$.

Conclusion Tissue Doppler velocities of different left ventricular (LV) walls ( $S^{\prime}, E^{\prime}, A^{\prime}$ of septal; $S^{\prime}, E^{\prime}$, of lateral; $S^{\prime}, A^{\prime}$ of inferior) in CAD at basal and after TMT were different from the no-CAD group, which were statistically different. Ratios such as $E / E^{\prime}, E / A^{\prime}$ of septal, lateral, and inferior, after TMT were useful for the detection of not only the presence of CAD but also for the severity of the CAD (for DVD: $12.29 \pm 2.09$ vs. $10.70 \pm$ 2.00, $p=0.023$; TVD: $12.2 \pm 2.19$ vs. $10.69 \pm 2.1, p=0.023$ ). In conclusion, diastolic stress evaluation is useful to predict the occurrence of CAD and its severity.
\end{abstract}

License terms 


\section{Introduction}

Coronary artery disease (CAD) is one of the leading causes of morbidity and mortality in the world and has neared its epidemic proportions. India has a similar scenario. Indian studies have revealed that cardiovascular diseases (CVDs) cause approximately $40 \%$ of deaths in urban areas and $30 \%$ of deaths in rural areas in India. ${ }^{1}$

Early detection of CAD among people with or without symptoms is the main task to reduce morbidity and mortality. There are various invasive and noninvasive methods to detect CAD among people with symptoms. Always invasive methods have their advantage and disadvantages, so noninvasive tests are low-cost modalities of CAD detection and are the need of the hour. Of these noninvasive modalities, various methods are used such as electrocardiogram (ECG), echocardiography (echo), CT, magnetic resonance imaging (MRI), and nuclear imaging.

Based on the concept that diastolic abnormalities occur earlier than symptoms, ECG changes, and systolic dysfunction during myocardial ischemia, diastolic stress test should identify ischemia earlier than another type of stress test methods. ${ }^{2}$ The authors want to study the usefulness of applying stress echo with treadmill test (TMT) for detection of CAD patients.

\section{Aims and Objectives}

The aim of this article is to measure changes in tissue Doppler imaging (TDI) parameters with exercise stress test in males and females and compare with the presence and severity of CAD on a coronary angiogram (CAG).

\section{Material and Methods}

\section{Procedure}

This study is conducted in our institution after approval by local ethical committee. Patients aged from 25 to 75 years who were presented to the outpatient clinic with chest pain or shortness of breath with normal left ventricular (LV) function and without acute coronary syndrome (ACS) were included. Exclusion criteria were patients who cannot perform TMT, patients with atrial fibrillation, patients with recent myocardial infarction/non-ST-segment elevation myocardial infarction / unstable angina (MI/NSTEMI/USA), more than stage 1 hypertension, chronic kidney disease, valvular heart disease, ECG presence of $\mathrm{Q}$ wave, with bundle branch pattern/intraventricular conduction delay (IVCD), and ejection fraction $(\mathrm{EF})<45 \%$.

The proforma of the study contained epidemiologic information (age, sex, occupation, and place), questionnaires for risk factor evaluation (smoking, alcohol, hypertension [HTN], diabetes mellitus, ischemic heart disease [IHD]), and information of clinical presentation (chest pain, dyspnea, etc.). Patients were subjected to routine ECG, 2D echo, and TMT as per requirement. TMT was performed with GE-T2100 CASE (GENERAL ELECTRIC COMPANY), version 6.7 machine using either Bruce or modified Bruce protocol.

Stress echo is performed using a standard ultrasound system (PHILIPS HD $11 \mathrm{XE}$; Philips) with a 1 - to $3-\mathrm{MHz}$ transducer during rest and at first minute of recovery. Wall motion analysis and mitral inflow parameter and TDI parameters were measured. In the apical four-chamber view pulse Doppler, sample volume was placed at mitral valve tips, and 5 to 10 cardiac cycles were recorded. The peak Doppler velocities of early (E) and late (A) mitral diastolic flow, deceleration time (DT), and E/A ratio were measured. ${ }^{3}$ Where there was a complete merging of $\mathrm{E}$ and $\mathrm{A}$ during the stress test, the resulting velocity was taken as E. TDI parameters were measured in basal septal, basal lateral and basal inferior wall, and in apical four- and apical two-chamber views. Myocardial velocities by TDI were measured at the septal and lateral mitral annular sites in the apical four-chamber view.

Coronary angiogram was done in all these patients. The presence of CAD was defined as $>50 \%$ luminal narrowing in at least one coronary vessel. The severity of CAD was classified as single-vessel diseases (SVDs), where there was > 50\% luminal narrowing one coronary vessel or double-vessel disease (DVD) in two coronary vessels, or triple-vessel diseases (TVDs) in all there coronary vessels. Left main disease was considered as DVD. The analysis was done to detect the important pulse wave and tissue Doppler velocity parameters for the detection of CAD and its severity with reference to males and females.

\section{Statistical Methods}

The descriptive statistics was done for all data collected and suitable statistical test used for comparison. Continuous variables are expressed as a mean + standard deviation (SD) and were analyzed using unpaired $t$-test and analysis of variance (ANOVA). Categorical variables were examined with the chi-square test with Yates correction. Statistical significance was taken for all correlation as $p<0.05$.

\section{Results}

In 51 study population, the mean age was $53.9 \pm 10.2$ years with a male:female::1.93:1. After CAG, 41 (80.4\%) patients had CAD and in 10 (19.6\%) patients no-CAD. Comparison of demographic features of CAD and no-CAD groups is depicted in - Table 1.

Presence of dyslipidemia and family history of CAD showed a tendency for the presence of CAD, but not the age, smoking, HTN, or diabetes. Gender distribution of the two groups is depicted in $\boldsymbol{- T a b l e} \mathbf{2}$ and $\boldsymbol{-}$ Fig. $\mathbf{1}$.

Comparison of demographic and laboratory parameters between the males and females showed that except for smoking, which was more in males, were comparable. CAD was more in males than females, which were statistically significant represented in - Table 3.

A 2D echo is done before starting the TMT and at the first minute after TMT. Fresh WMA (wall motion abnormalities) were noted. All pulse wave and tissue Doppler-derived parameters, before and after TMT, were mentioned in - Table 4. E, A velocity, E/A ratio, and DT on pulse wave Doppler before starting the TMT and also after TMT were same in both the groups (CAD and no-CAD). 
Table 1 Demographic features of both groups

\begin{tabular}{|l|l|l|l|}
\hline Characteristics & CAD & No CAD & $p$ Value \\
\hline Age $(y)$ & $53.88 \pm$ & $49.90 \pm$ & 0.97 \\
& 10.22 & 7.85 & \\
\hline Smoker & $18(43.9 \%)$ & $3(30 \%)$ & 0.43 \\
\hline Hypertension & $15(36.59)$ & $4(40 \%)$ & 0.84 \\
\hline Diabetes & $17(41.46 \%)$ & $4(40 \%)$ & 0.93 \\
\hline Family history of CAD & $17(41.46 \%)$ & $1(10 \%)$ & 0.08 \\
\hline Hypercholesterolemia & $17(41.46 \%)$ & $0(0)$ & 0.07 \\
\hline
\end{tabular}

Abbreviation: CAD, coronary artery disease.

Table 2 Gender distribution of the study population

\begin{tabular}{|l|l|l|l|l|}
\hline Gender & CAD & $\%$ & No-CAD & $\%$ \\
\hline Male & 27 & 65.85 & 4 & 40 \\
\hline Female & 14 & 34.15 & 6 & 60 \\
\hline Total & 41 & 100 & 10 & 100 \\
\hline$p$ Value & 0.1627 &
\end{tabular}

Abbreviation: CAD, coronary artery disease.

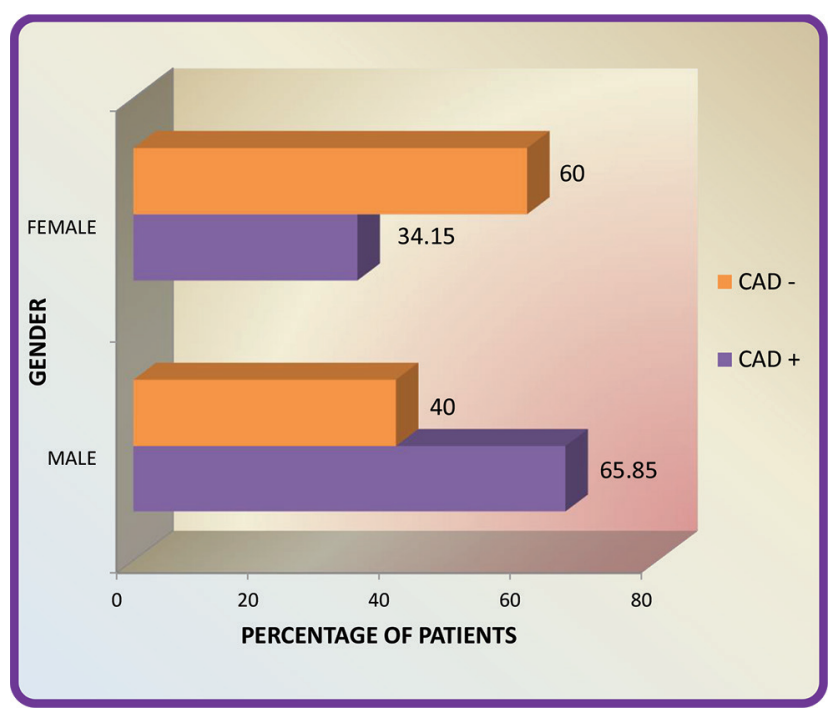

Fig. 1 Gender distribution among study groups.

However, tissue Doppler velocities were different in CAD and no-CAD patients in both at rest and after TMT - Table 5.

Tissue Doppler velocities, $S^{\prime}$ septal, E' septal, $A^{\prime}$ septal, $S^{\prime}$ lateral, $E^{\prime}$ lateral, $S^{\prime}$ inferior, $A^{\prime}$ inferior ratios, $E / E^{\prime}$ septal, $E / A^{\prime}$ septal, $E / E^{\prime}$ lateral, $E^{\prime} / A$ lateral, $E / E^{\prime}$ inferior, and $E^{\prime} / A^{\prime}$ inferior
Table 3 Compares the demographic, laboratory, and CAG findings in males versus females

\begin{tabular}{|l|l|l|l|}
\hline Characteristics & Males (31) & Females (20) & $p$ Value \\
\hline Average age & $54.25 \mathrm{y}$ & $53.9 \mathrm{y}$ & \\
\hline HTN & $14(45.16 \%)$ & $10(50 \%)$ & 0.348 \\
\hline DM & $11(35.48 \%)$ & $10(50 \%)$ & 0.806 \\
\hline Smoking & $20(64.5 \%)$ & $0(0 \%)$ & 0.000 \\
\hline Family h/o CAD & $10(32.25 \%)$ & $8(40 \%)$ & 0.603 \\
\hline CAD & $28(90.3 \%)$ & $13(65 \%)$ & 0.001 \\
\hline SVD & $14(45.16 \%)$ & $6(30 \%)$ & 0.042 \\
\hline DVD & $5(16.12)$ & $2(10 \%)$ & 0.237 \\
\hline TVD & $8(25.8 \%)$ & $5(25 \%)$ & 0.371 \\
\hline Average TC & 230.9 & 216.45 & 0.182 \\
\hline Average LDL & 151.61 & 142.4 & 0.357 \\
\hline $\begin{array}{l}\text { Average } \\
\text { non-HDLC }\end{array}$ & 189.90 & 175.65 & 0.220 \\
\hline Average BMI & 23.9 & 23.65 & 0.720 \\
\hline
\end{tabular}

Abbreviations: BMI, body mass index; CAD, coronary artery disease; CAG, coronary angiogram; DM, diabetes mellitus; DVD, double-vessel disease; HDLC, high-density lipoprotein cholesterol; HTN, hypertension; SVD, single-vessel disease; TVD, triple-vessel disease.

were significantly different even at basal echo time, which were more significant during exercise (-Table 6). A' lateral and $\mathrm{E}^{\prime}$ inferior were not different at basal and after stress in between the groups.

The tissue Doppler velocities ratios during TMT, but not the velocities as such, were helpful in detecting the severity of CAD ( - Table 7$)$.

Treadmill test positive had $56 \%$ sensitivity for detection of CAD. Along with stress echo its sensitivity was $71 \%$. By including WMA with other parameters, sensitivity for detection of CAD was $75 \%$.

Even though univariate analysis showed many TDI parameters were useful in detection and severity of CAD, multivariate regression analysis did not indicate that. Multivariate regression analysis showed that positive TMT had a tendency for detection of CAD, whereas the presence of WMA after exercise was determinant for CAD ( - Table 8).

\section{Discussion}

Tissue Doppler examination during the stress may detect the diastolic and systolic dysfunctions due to stress-induced ischemia. Evidence has been piling on the importance of

Table 4 Comparison of pulse wave Doppler parameters before and after in both study groups

\begin{tabular}{|l|l|l|l|l|l|l|}
\hline \multirow{2}{*}{ Pulse wave Doppler parameter } & \multicolumn{3}{|c|}{ Rest } & \multicolumn{3}{c|}{ After TMT } \\
\cline { 2 - 7 } & CAD & No-CAD & $p$ Value & CAD & No-CAD & $p$ Value \\
\hline E (cm/s) & $0.76 \pm 0.18$ & $87.60 \pm 22.36$ & 0.2903 & $1.03 \pm 0.22$ & $1.11 \pm 0.21$ & 0.3 \\
\hline A (cm/s) & $0.85 \pm 0.21$ & $1.03 \pm 12.84$ & 0.3350 & $1.03 \pm 0.2$ & $0.99 \pm 0.1$ & 0.3 \\
\hline E/A & $0.94 \pm 0.29$ & $0.86 \pm 0.30$ & 0.097 & $1.01 \pm 0.24$ & $1.12 \pm 0.16$ & 0.097 \\
\hline DT (ms) & $215.78 \pm 33.21$ & $215.80 \pm 33.21$ & 0.17 & $193.61 \pm 442.80$ & $181.10 \pm 18.50$ & 0.17 \\
\hline
\end{tabular}

Abbreviations: CAD, coronary artery disease; DT, declaration time. 
Table 5 TDI parameters in both groups

\begin{tabular}{|l|l|l|l|l|l|l|}
\hline \multirow{2}{*}{$\begin{array}{l}\text { Tissue Doppler } \\
\text { parameter }\end{array}$} & \multicolumn{2}{l|}{ Rest } & \multicolumn{2}{l|}{ After TMT } \\
\cline { 2 - 7 } & CAD & No-CAD & $p$ Value & CAD & No-CAD & $p$ Value \\
\hline Average $S^{\prime}(\mathrm{cm} / \mathrm{s})$ & $6.25 \pm 1.24$ & $8.11 \pm 1.63$ & 0.0014 & $7.06 \pm 1.49$ & $9.74 \pm 1.92$ & 0.0014 \\
\hline Average $E^{\prime}(\mathrm{cm} / \mathrm{s})$ & $8.20 \pm 1.87$ & $10.47 \pm 2.41$ & 0.0020 & $8.58 \pm 2.17$ & $12.19 \pm 2.17$ & 0.0020 \\
\hline Average $A^{\prime}(\mathrm{cm} / \mathrm{s})$ & $7.37 \pm 1.81$ & $8.75 \pm 1.62$ & 0.0182 & $8.00 \pm 2.02$ & $9.85 \pm 1.95$ & 0.0182 \\
\hline Average $\mathrm{E} / \mathrm{E}^{\prime}(\mathrm{cm} / \mathrm{s})$ & $9.37 \pm 0.99$ & $8.37 \pm 0.98$ & $<0.00001$ & $12.52 \pm 1.85$ & $9.29 \pm 1.21$ & $<0.00001$ \\
\hline
\end{tabular}

Abbreviations: CAD, coronary artery disease; TDI, tissue Doppler imaging; TMT, treadmill test.

Table 6 All walls TDI parameters in CAD and no-CAD patients

\begin{tabular}{|c|c|c|c|c|c|c|}
\hline \multirow[t]{2}{*}{ TDI parameters } & \multicolumn{3}{|l|}{ Rest } & \multicolumn{3}{|l|}{ After TMT } \\
\hline & CAD & No-CAD & $p$ Value & CAD & No-CAD & $p$ Value \\
\hline $\mathrm{S}^{\prime}$ septal $(\mathrm{cm} / \mathrm{s})$ & $6.00 \pm 1.22$ & $7.74 \pm 1.62$ & 0.002 & $6.84 \pm 1.42$ & $9.57 \pm 2.07$ & 0.002 \\
\hline$E^{\prime}$ septal $(\mathrm{cm} / \mathrm{s})$ & $7.87 \pm 1.81$ & $10.10 \pm 2.26$ & 0.002 & $8.14 \pm 2.16$ & $11.98 \pm 2.78$ & 0.002 \\
\hline $\mathrm{A}^{\prime}$ septal $(\mathrm{cm} / \mathrm{s})$ & $7.18 \pm 1.68$ & $8.63 \pm 1.66$ & 0.02 & $7.83 \pm 1.99$ & $9.85 \pm 2.18$ & 0.02 \\
\hline E/E' septal & $9.72 \pm 1.07$ & $8.60 \pm 0.82$ & $<0.0001$ & $13.15 \pm 2.23$ & $9.46 \pm 1.19$ & $<0.0001$ \\
\hline E/A' septal & $1.10 \pm 0.18$ & $1.18 \pm 0.17$ & 0.002 & $1.04 \pm 0.15$ & $1.21 \pm 0.13$ & 0.002 \\
\hline S' lateral $(\mathrm{cm} / \mathrm{s})$ & $6.84 \pm 1.42$ & $9.03 \pm 1.78$ & 0.002 & $7.51 \pm 1.83$ & $10.51 \pm 2.21$ & 0.002 \\
\hline $\mathrm{E}^{\prime}$ lateral $(\mathrm{cm} / \mathrm{s})$ & $8.79 \pm 2.15$ & $11.39 \pm 2.55$ & 0.002 & $9.02 \pm 2.52$ & $13.13 \pm 3.01$ & 0.002 \\
\hline $\mathrm{A}^{\prime}$ lateral $(\mathrm{cm} / \mathrm{s})$ & $7.62 \pm 2.29$ & $9.06 \pm 2.15$ & 0.03 & $8.29 \pm 2.37$ & $10.01 \pm 1.96$ & 0.03 \\
\hline E/E' lateral & $8.78 \pm 1.10$ & $7.69 \pm 1.12$ & $<0.0001$ & $11.99 \pm 2.12$ & $8.64 \pm 1.19$ & $<0.0001$ \\
\hline$E^{\prime} / A^{\prime}$ lateral & $1.22 \pm 0.36$ & $1.29 \pm 0.36$ & 0.004 & $1.09 \pm 0.18$ & $1.31 \pm 0.17$ & 0.004 \\
\hline $\mathrm{S}^{\prime}$ inferior $(\mathrm{cm} / \mathrm{s})$ & $5.93 \pm 1.17$ & $7.59 \pm 1.63$ & 0.001 & $6.84 \pm 1.47$ & $9.13 \pm 1.59$ & 0.001 \\
\hline $\mathrm{E}^{\prime}$ inferior $(\mathrm{cm} / \mathrm{s})$ & $7.97 \pm 1.85$ & $9.93 \pm 2.52$ & 0.24 & $8.56 \pm 2.24$ & $22.60 \pm 35.36$ & 0.24 \\
\hline $\mathrm{A}^{\prime}$ inferior $(\mathrm{cm} / \mathrm{s})$ & $7.29 \pm 1.70$ & $8.56 \pm 1.59$ & 0.02 & $7.90 \pm 1.87$ & $9.73 \pm 2.04$ & 0.02 \\
\hline$E / E^{\prime}$ inferior & $9.63 \pm 1.27$ & $8.85 \pm 1.19$ & 0.0001 & $12.44 \pm 2.18$ & $9.81 \pm 1.37$ & 0.0001 \\
\hline$E^{\prime} / A^{\prime}$ inferior & $1.10 \pm 0.19$ & $1.15 \pm 0.16$ & 0.11 & $1.08 \pm 0.16$ & $1.16 \pm 0.12$ & 0.11 \\
\hline
\end{tabular}

Abbreviations: CAD, coronary artery disease; TDI, tissue Doppler imaging.

Table 7 TDI parameters versus severity of CAD

\begin{tabular}{|l|l|l|l|l|l|l|}
\hline \multirow{2}{*}{ CAD } & \multicolumn{4}{l|}{ Average $E / E^{\prime}$ at baseline } & \multicolumn{4}{l|}{ Average $E / E^{\prime}$ after exercise } \\
\cline { 2 - 7 } & CAD & No-CAD & $p$ Value & CAD & No-CAD & $p$ Value \\
\hline SVD & $9.29 \pm 1.00$ & $8.83 \pm 1.18$ & 0.2 & $12.18 \pm 1.89$ & $11.03 \pm 2.71$ & 0.2 \\
\hline DVD & $9.3 \pm 1$ & $8.8 \pm 1.1$ & 0.3 & $12.29 \pm 2.09$ & $10.70 \pm 2.00$ & 0.02 \\
\hline TVD & $9.27 \pm 1.03$ & $8.9 \pm 1.12$ & 0.3 & $12.2 \pm 2.19$ & $10.69 \pm 2.1$ & 0.02 \\
\hline
\end{tabular}

Abbreviations: CAD, coronary artery disease; DVD, double-vessel disease; SVD, single-vessel disease; TVD, triple-vessel disease.

physiological assessment rather than morphologic assessment for CAD detection. Regional stunning due to myocardial ischemia can be diagnosed by tissue Doppler measurements. The myocardial ischemia can also be detected by measurement of reduced transmyocardial velocity. Regional diastolic dysfunction can be detected and measured by TDI, so TDI parameters such as systolic velocity $\left(S^{\prime}\right)$, early diastolic velocity $\left(E^{\prime}\right)$, and late diastolic velocity $\left(A^{\prime}\right)$ can reflect changes during ischemia. Changes documented during ischemia are reduced and delayed peak systolic and diastolic velocities, reduced myocardial velocity gradients, and diastolic dysfunction, which is demonstrated in various studies. Because diastolic dysfunction may persist after recovery of normal systolic function, after short time of coronary occlusion by balloon during coronary angioplasty, ${ }^{3}$ in post treadmill exercise stress, echo can identify diastolic dysfunction immediately after cessation of exercise.

Baseline TDI systolic velocity $\left(S^{\prime}\right)$ was lower in CAD patients than without CAD (6.25 vs. $8.11 \mathrm{~cm} / \mathrm{s})$ as shown in other studies. This study had lower $E^{\prime}$ and $A^{\prime}$ in CAD patients. However, $E / E^{\prime}$ slightly higher than normal value in CAD group (9.37 vs. 8.37). Tissue Doppler systolic velocity 
Table 8 Multivariate regression analysis

\begin{tabular}{|l|l|l|l|}
\hline Variable & Odds ratio & $\mathbf{9 5 \%} \mathrm{Cl}$ & $\mathbf{p}$ Value \\
\hline Smoking & 1.83 & $0.41-8.07$ & 0.43 \\
\hline $\begin{array}{l}\text { Hypercholesterol- } \\
\text { emia }\end{array}$ & 15.00 & $0.82-273.34$ & 0.07 \\
\hline Hypertension & 0.86 & $0.21-3.57$ & 0.84 \\
\hline Diabetes & 1.06 & $0.26-4.35$ & 0.93 \\
\hline Positive TMT & 1.33 & $0.29-6.13$ & 0.07 \\
\hline $\begin{array}{l}\text { Presence of WMA } \\
\text { after exercise }\end{array}$ & 71.84 & $3.84-1342.39$ & 0.004 \\
\hline $\begin{array}{l}\text { Increase in E/E' } \\
\text { Average after } \\
\text { exercise }\end{array}$ & 3.17 & $0.03-10.96$ & 0.25 \\
\hline
\end{tabular}

Abbreviations: $\mathrm{Cl}$, confidence interval; TMT, treadmill test; WMA, wall motion abnormality.

for different segments in baseline mainly basal septal, basal lateral, and basal inferior are 8.11,10.47, and $8.75 \mathrm{~cm} / \mathrm{s}$, respectively. There were significant changes of tissue Doppler echo parameters in patients with CAD after stress test. The systolic velocity did not increase after stress test in CAD patients.

Hoffmann et $\mathrm{al}^{4}$ have studied 82 patients with suspected angina pectoris, no previous cardiac history, and normal ejection fraction. All the patients were examined with color TDI before CAG, at baseline. The baseline TDI parameters in patients with and without CAD compared with this study (-Table 9).

As shown above, there is a statistically significant difference in average $S^{\prime}$ and average $E / E^{\prime}$ between patients with and without CAD whereas difference in average $E^{\prime}$ and average $A^{\prime}$ is statistically insignificant. In this study in the CAD group, the average $S^{\prime}$, average $E^{\prime}$, and average $A^{\prime}$ along with the average $\mathrm{E} / \mathrm{E}^{\prime}$ were significantly lower values than in no-CAD group, which were statistically significant. The increase in $\mathrm{E} / \mathrm{E}^{\prime}$ ratio did not correlate with SVD but well with DVD and TVD.

In the study by Nitta et $a{ }^{5}$, the hypothesis that, in patients with nonobstructive CAD documented by CAG, the index of LV diastolic function from ECG-gated SPECT is useful in detecting myocardial ischemia, was tested. Multivariate logistic regression analysis showed that peak filling rate (PFR) at redistribution was an independent predictor of the presence of myocardial ischemia in patients with nonobstructive
CAD, by angiogram (odds ratio: $0.15,95 \%$ confidence interval: 0.04-0.51, $p=0.002$ ). Summed difference scores (SDS) in patients with myocardial ischemia and those without were $3.5 \pm 1.8$ and $0.4 \pm 0.5$, respectively $(p<0.001)$. PFR after stress was significantly lower in patients with myocardial ischemia than in those without. Lower PFRs after stress and at redistribution were inversely correlated with SDS. PFR was correlated with mitral annular velocity on tissue Doppler echo, which is widely used as an index of LV diastolic function in the clinical setting.

In the MYDISE ${ }^{6}$ (Myocardial Doppler in Stress Echocardiography) study, Mädler et., al have studied tissue Doppler digital data during dobutamine stress in 289 patients, and measured myocardial responses of $11 \mathrm{LV}$ segments, by offline analysis. Ninety-two normal patients were compared with 48 patients with coronary disease, in a prospective series of 149 patients referred with chest pain. Optimal diagnostic accuracy was achieved by logistic regression models, using systolic velocities at maximal stress in seven myocardial segments, adjusting for independent correlations directly with heart rate and inversely with age and female gender (all $p<0.001$ ). They have demonstrated that CAD can be diagnosed accurately and objectively, from off-line measurements of myocardial velocities recorded noninvasively by tissue Doppler echocardiography during dobutamine stress.

In this study, difference in average $S^{\prime}$ and average $E^{\prime}$ among two study groups is statistically insignificant, but difference in average $\mathrm{A}^{\prime}$ and $\mathrm{Avg} \mathrm{E} / \mathrm{E}^{\prime}$ has significant $p$ value. The diastolic parameters $\mathrm{E}$ prime and A prime were not competitively increased in CAD group. However, there were decrease in $\mathrm{E}$ velocity and increase in A velocity as occurred in other studies. Average E/E' ratios of all three myocardial segments measured are significantly increased and the increasing change (3.15) well correlated with CAD detection in diastolic stress test. The average increase in $\mathrm{E} / \mathrm{E}^{\prime}$ ratio well correlated in patients with positive TMT.

In this study, TMT positive had $56 \%$ sensitivity for detection of CAD, when combining an increase in $E / E^{\prime}$ value with stress echo, its sensitivity rose to $71 \%$. Moreover including objective measurement of WMAs with other parameters sensitivity for detection of CAD was as high as $75 \%$ in this study. It needs further study with a larger population to decide whether $\mathrm{E} / \mathrm{E}^{\prime}$ stands as a sensitive marker to diagnose CAD.

\section{Conflict of Interest}

None.

Table 9 Comparison of TDI parameters in study by Hoffmann et al and this study

\begin{tabular}{|l|l|l|l|l|l|l|}
\hline \multirow{2}{*}{ TDI parameters at rest } & \multicolumn{5}{l}{ Study by Soren Hoffmann et al (82 patients) } & \multicolumn{2}{l|}{ This study (51 patients) } \\
\cline { 2 - 8 } & CAD & No-CAD & $p$ Value & CAD + & CAD - & $p$ Value \\
\hline Average $S^{\prime}(\mathrm{cm} / \mathrm{s})$ & $5.58+0.96$ & $6.06+0.89$ & $0.008^{*}$ & $6.25 \pm 1.24$ & $8.11 \pm 1.63$ & 0.001 \\
\hline Average $E^{\prime}(\mathrm{cm} / \mathrm{s})$ & $6.31+1.65$ & $6.78+1.46$ & NS & $8.20 \pm 1.87$ & $10.47 \pm 2.41$ & 0.002 \\
\hline Average $A^{\prime}(\mathrm{cm} / \mathrm{s})$ & $7.16+1.53$ & $7.16+1.32$ & NS & $7.37 \pm 1.81$ & $8.75 \pm 1.62$ & 0.02 \\
\hline Average $E / E^{\prime}$ & $11.6+3.99$ & $10.4+2.66$ & 0.032 & $9.37 \pm 0.99$ & $8.37 \pm 0.98$ & $<0.00001$ \\
\hline
\end{tabular}

Abbreviations: CAD, coronary artery disease; NS, nonsignificant; TDI, tissue Doppler imaging. 


\section{References}

1 Gupta R. Recent trends in coronary heart disease epidemiology in India. Indian Heart J 2008;60(2 Suppl B):B4-B18

2 Nesto RW, Kowalchuk GJ. The ischemic cascade: temporal sequence of hemodynamic, electrocardiographic and symptomatic expressions of ischemia. Am J Cardiol 1987; 59(7):23C-30C

3 Mulvagh S, Quiñones MA, Kleiman NS, Cheirif J, Zoghbi WA. Estimation of left ventricular end-diastolic pressure from Doppler transmitral flow velocity in cardiac patients independent of systolic performance. J Am Coll Cardiol 1992;20(1):112-119

4 Hoffmann S, Mogelvang R, Olsen NT, et al. Tissue Doppler echocardiography reveals distinct patterns of impaired myocardial velocities in different degrees of coronary artery disease. Eur J Echocardiogr 2010;11(6):544-549
5 Nitta K, Kurisu S, Sumimoto Y, et al. Diagnostic value of peak filling rate derived from ECG-gated myocardial perfusion SPECT for detecting myocardial ischaemia in patients with non-obstructive coronary artery disease. Acta Cardiol 2019;:1-5. doi: 10.1080/00015385.2018.1544698. [Epub ahead of print]

6 Mädler CF, Payne N, Wilkenshoff U, et al; Myocardial Doppler in Stress Echocardiography (MYDISE) Study Investigators. Non-invasive diagnosis of coronary artery disease by quantitative stress echocardiography: optimal diagnostic models using off-line tissue Doppler in the MYDISE study. Eur Heart J 2003;24(17):1584-1594 